\title{
Downregulation of CIP2A inhibits cancer cell proliferation and vascularization in renal clear cell carcinoma
}

\author{
Hui Gao, Yuqiao Li, Tao Lin, Yan Cheng, Yunbo Ma
}

Background. CIP2A has been proved to play a role as an oncogene in various types of malignancies while its functionality in renal clear cell carcinoma has not been investigated. Our study aimed to investigate the role of CIP2A in renal clear cell carcinoma and to explore the possible mechanisms.

Methods. A total of 80 patients with renal clear cell carcinoma and 32 healthy people were included in the study. Expression of CIP2A was detected by qRT-PCR. CIP2A silencing renal clear cell carcinoma cell line was established. Its effects on cell proliferation and migration were verified by CCK-8 assay and Transwell cell assay, respectively. The effects of CIP2A overexpression on AKT and VEGF were investigated.

Results. CIP2A expression level was increased in tumor tissues compared to adjacent healthy tissues. Serum levels of CIP2A protein were higher in cancer patients than in healthy controls, and serum levels of CIP2A protein were increased with increased stage of primary tumor. Serum CIP2A protein can be used to accurately predict renal clear cell carcinoma and its prognosis. CIP2A siRNA silencing inhibited tumor cell proliferation, and treatment with Akt activator reduced this inhibitory effect. CIP2A siRNA silencing decreased the expression level of VEGF and phosphorylation levels of AKT in renal clear cell carcinoma cells, while AKT activator treatment showed no significant effects on CIP2A expression.

Conclusion. Downregulation of CIP2A can inhibit cancer cell proliferation and vascularization in renal clear cell carcinoma through inactivation of the Akt pathway and its downstream VEGF.

Key words: renal clear cell carcinoma, CIP2A, AKT, VEGF

Received: October 30, 2018; Accepted with revision: June 26, 2019; Available online: August 21, 2019

https://doi.org/10.5507/bp.2019.031

(c) 2020 The Authors; https://creativecommons.org/licenses/by/4.0/

Department of Urology, Liaocheng People's Hospital, Liaocheng City, Shandong Province, P. R. China, 252000

Corresponding author: Yunbo Ma, e-mail: mayunbo3@163.com

\section{BACKGROUND}

Renal cell carcinoma is a type of malignant tumor that originates form the lining of the proximal convoluted tubule, which is a component of the very small tubes that transport waste from the blood circulatory system to urine ${ }^{1}$. As one of the leading causes of cancer-related death, renal cell carcinoma causes more than 15,000 deaths every year world wide ${ }^{2}$. Renal cell carcinoma can generally be divided into four histological types including renal clear cell carcinoma, renal oncocytoma, papillary renal cell carcinoma and chromophobe renal cell carcinoma $^{3}$. As the dominant type of renal cell carcinoma, renal clear cell carcinoma accounts for about $80 \%$ of all cases $^{4,5}$. The occurrence of renal clear cell carcinoma has been found to mainly correlate with mutations of tumor suppressor genes ${ }^{6,7}$. However, the involvement of the oncogene in the pathogenesis of renal clear cell carcinoma still has not been well studied. Cancerous inhibitor of PP2A, a.k.a. CIP2A, is a type of trimeric serine-threonine phosphatase that plays pivotal roles in both normal physiological processes and pathological processes such as cancer maintenance and progression by dephosphorylating a variety of phosphorylated cellular serine/threonine proteins ${ }^{8}$. CIP2A is usually upregulated in cancers, such as lung cancer ${ }^{9}$ and gastric cancer ${ }^{10}$ to mediate cancer cell proliferation, migration and invasion, and inhibition of CIP2A is considered as a potential therapeutic target for cancer therapy ${ }^{10}$. It has been reported that CIP2A expression is closely correlated with the development and progression of renal cell carcinoma ${ }^{11}$, while the specific role in renal clear cell carcinoma as well as the molecular function mechanism is still unclear. Therefore, our study investigated the signal transduction of CIP2A in renal clear cell carcinoma. Our study provided references for future studies on the pathogenesis on this disease.

\section{MATERIALS AND METHODS}

\section{Subjects}

A total of 80 patients ( 46 males and 34 females) with renal clear cell carcinoma were enrolled in Liaocheng People's Hospital (a first-class hospital, Shandong, China) from June 2011 to June 2012. The patients aged from 22 to 71 years, with a mean age of $46.7 \pm 7.2$ years. All patients were Han Chinese. Pathological and imaging tests were performed on these patients to confirm renal clear cell carcinoma. Patients with other renal diseases and other types of malignancies were excluded. Primary tumor staging was performed according to the following standards: greatest dimension $<7 \mathrm{~cm}$, and tumor was only in the kidney, cT1; greatest dimension $>7 \mathrm{~cm}$, and tumor was only in the kidney, cT2; tumor invaded major vein tis- 
sue around the kidney but there was no invasion into the adrenal gland (on top of the kidney) or beyond Gerota's fascia, cT3; tumor has spread beyond Gerota's fascia or invaded into the adrenal gland, cT4. There were 18 cases in cT1, 21 in cT2, 20 in cT3 and 21 in cT4.Tumor tissues and adjacent healthy tissues with $5 \mathrm{~cm}$ around the tumor were collected during surgery and were confirmed pathologically. A total of 34 healthy people and similar gender and age distributions were also enrolled at the same time to serve as controls. The control group included 20 males and 14 females, and aged from 24 to 70 years, with a mean age of $47.4 \pm 7.9$ years. The ethics committee of Liaocheng People's Hospital approved this study. All participants signed informed consent.

\section{Cell lines and cell culture}

Human renal clear cell carcinoma Caki-1 was purchased from ATCC. Cells were cultured in ATCCformulated McCoy's 5a Medium (Catalog No. 30-2007, ATCC) that supplemented with $10 \%$ fetal bovine serum in an incubator at $37{ }^{\circ} \mathrm{C}(5 \% \mathrm{CO} 2)$. Cells were collected during logarithmic growth phase for subsequent experiments.

\section{Establishment of CIP2A siRNA silencing renal clear cell carcinoma cell lines}

CIP2A siRNA (Catalog \# AM16708) and Silencer®) Negative Control \#1 siRNA (Catalog \# AM4611) were purchased from Thermo Fisher Scientific. Lipofectamine 2000 reagent (cat. no. 11668-019; Invitrogen, Thermo Fisher Scientific, Inc.) was used to transfect $50 \mathrm{nM}$ siRNA into $6 \times 10^{5}$ cells. After transfection, cells were cultured in RPMI-1640 medium (Hyclone; GE Healthcare Life Sciences, Logan, UT, USA) for $48 \mathrm{~h}$ at $37^{\circ} \mathrm{C}$ before subsequent experiments.

Blood extraction and detection of serum CIP2A by ELISA

In the morning of the day just after admission, $25 \mathrm{~mL}$ fasting blood was extracted from each participant. Blood was kept for $3 \mathrm{~h}$ at room temperature and centrifuged at $1875 \mathrm{rpm}$ for $15 \mathrm{~min}$ to separate serum. Serum levels of CIP2A were measured using an ELISA Kit (MyBioSource, CA, USA) according to the instructions. Optical density at $450 \mathrm{~nm}$ was measured using an automated microplate reader (Thermo Fisher Scientific, Waltham, MA, USA). Serum levels of CIP2A were normalized to $\mathrm{ng} / \mathrm{mL}$.

\section{Cell proliferation assay}

Cell suspension was prepared using cells collected during logarithmic growth phase, and $100 \mu \mathrm{L}$ of cell suspension containing $4 \times 10^{3}$ cells was added into each well of 96-well plate. Then $10 \mathrm{uL}$ of CCK-8 solution was added 24 , 48, 72 and 96 h later. After the addition of CCK-8 solution, Fisherbrand ${ }^{\mathrm{TM}}$ accuSkan ${ }^{\mathrm{TM}}$ GO UV/Vis Microplate Spectrophotometer was used to measured optical density at $450 \mathrm{~nm}$ (Fisher Scientific) after another 4 hours' cell culture.

\section{Real-time quantitative PCR}

Tumor tissues and healthy tissues were ground in liquid nitrogen, and Trizol reagent (Invitrogen, USA) was added to extract total RNA. Trizol reagent was also mixed with in vitro cultured cells to extract total RNA. Total RNA extracted was repeated until all RNA samples showed an A260/A280 ratio between 1.8 and 2.0 (NanoDrop ${ }^{\text {TM }} 2000$ Spectrophotometers, Thermo Fisher Scientific, USA). RNA samples were subjected to reverse transcription to synthesize cDNA. SYBR $®$ Green Real-Time PCR Master Mixes (Thermo Fisher Scientific, USA) was used to prepare reaction system. Following primers were used in PCR reactions: 5'-TGCGGCACTTGGAGGTAATTTC-3' (forward) and 5'-AGCTCTACAAGGCAACTCAAGC-3' (reverse) for CIP2A; 5'-GACCTCTATGCCAACACAGT-3' (forward) and 5'-AGTACTTGCGCTCAGGAGGA-3' (reverse) for $\beta$-actin. PCR reaction conditions were: 95 ${ }^{\circ} \mathrm{C}$ for $55 \mathrm{~s}$, followed by 40 cycles of $95{ }^{\circ} \mathrm{C}$ for $20 \mathrm{~s}$ and $60{ }^{\circ} \mathrm{C}$ for $42 \mathrm{~s}$. Data were processed using $2^{-\Delta \Delta \mathrm{CT}}$ method, and relative expression level of CIP2A was normalized to endogenous control $\beta$-actin.

\section{Western-blot}

RIPA solution (Thermo Fisher Scientific, USA) was adopted to extract total protein from in vitro cultured cells, and protein quantification was performed by BCA method. Then 10\% SDS-PAGE gel electrophoresis was carried out using $20 \mu \mathrm{g}$ protein from each sample. After transmembrane, blocking was performed by incubating PVDF membranes with 5\% skimmed milk at room temperature for $2 \mathrm{~h}$. After that, membranes were washed with PBS 3 times, $5 \mathrm{~min}$ for each time, followed by incubation with primary antibodies including rabbit anti-p-Akt (1: 2000, ab38449, Abcam), anti-Akt (1: 2000, ab8805, Abcam), anti-VEGF (1: 2000, ab46153, Abcam), anti-CIP2A (1:1000, ABC307, EMD Millipore) and antiGAPDH primary antibody (1: 1000, ab8245, Abcam) overnight at $4{ }^{\circ} \mathrm{C}$. After that, membranes were washed 3 times with PBS, 5 min each time, which was followed by incubation with anti-rabbit IgG-HRP secondary antibody (1:1000, MBS435036, MyBioSource) at room temperature for $2 \mathrm{~h}$. Signal development was performed using ECL (Sigma-Aldrich, USA) method and signals were detected by MYECL ${ }^{\mathrm{TM}}$ Imager (Thermo Fisher Scientific, USA). Relative expression levels of p-Akt, Akt, VEGF and CIP2A were normalized to endogenous control GAPDH using image $\mathrm{J}$ software.

\section{Statistical analysis}

All data were processed using SPSS19.0 (SPSS Inc., USA) was used. Count data were compared by the chisquare test. Measurement data were expressed as ( $\mathrm{X} \pm \mathrm{sd})$, and $\mathrm{t}$ tests were used for comparisons between two groups, and analysis of variance and LSD test were used for comparisons among multiple groups. $P<0.05$ indicate a difference that was statistically significant. 


\section{RESULTS}

Expression of CIP2A in tumor tissues and adjacent healthy tissues of 80 patients with renal clear cell carcinoma

Differential expression of certain genes between tumor tissues and adjacent healthy tissues indicates their involvement in the pathogenesis of cancer. Fig. 1 shows the expression of CIP2A in tumor tissues and adjacent healthy tissues of 80 patients with renal clear cell carcinoma. qRT-PCR was carried to measure the expression level of CIP2A for renal clear cell carcinoma. As shown in Fig. 1, significantly higher expression level of CIP2A mRNA in tumor tissues than that in adjacent healthy tissues was found in 59 out of 80 patients $(P<0.05)$. In contrast, significantly lower expression level of CIP2A mRNA in tumor tissues than that in adjacent healthy tissues was only found in 6 cases $(P<0.05)$. No significant difference was found in 15 cases $(P>0.05)$. The results suggest that upregulation of CIP2A is very likely to be involved in the pathogenesis of renal clear cell carcinoma.

\section{Serum levels of CIP2A in renal clear cell carcinoma} patients and its diagnostic values

ELISA was performed to measure serum levels of CIP2A in both renal clear cell carcinoma patients and healthy controls. As shown in Fig. 2a, serum levels of CIP2A in patients with different stages of renal clear cell carcinoma patients were significantly higher than in healthy controls $(P<0.05)$. In addition, serum levels of CIP2A were increased with the increase of stage of primary tumor $(P<0.05)$. ROC curve analysis was performed to evaluate the diagnostic value of serum CIP2A for different stages of renal clear cell carcinoma with renal clear cell carcinoma patients as true positive cases and healthy controls as true negative cases. The area under the curve (AUC) of serum CIP2A in the diagnosis of cT1 stage patients was 0.8660 with $95 \%$ confidence interval of 0.7688 to 0.9632 ( $P<0.0001$, Fig. 2b). The AUC of serum CIP2A in the diagnosis of cT2 patients was 0.9762 with $95 \%$ confidence interval of 0.9438 to 1.000 ( $P<0.0001$, Fig. 2c), in the diagnosis of cT3 patients was 0.9816 with $95 \%$ con- fidence interval of 0.9550 to 1.008 ( $P<0.0001$, Fig. 2 d), and in the diagnosis of cT4 patients was 0.9825 with $95 \%$ confidence interval of 0.9575 to 1.007 ( $P<0.0001$, Fig. 2e).

\section{Prognostic value of serum CIP2A for renal clear cell carcinoma}

The 80 patients with renal clear cell carcinoma were divided into a high expression group $(n=40)$ and a low expression group $(n=40)$ according to the median serum level of CIP2A protein. All patients were followed up for 5 years or until their deaths. Survival curves were plotted based on the survival conditions that collected during follow-up using Kaplan-Meier method and were compared by $\log$ rank t test. Results showed that the survival rate of patients with high serum level of CIP2A protein was significantly lower than that of patients with high serum level of CIP2A protein $(P<0.0001$, Fig. 3$)$. In addition, serum level of CIP2A protein in patients with renal clear cell carcinoma was not significantly correlated with patients' age, gender or living habits including smoking and drinking (Table 1).

\section{CIP2A is likely an upstream activator of Akt pathway and VEGF}

CIP2A siRNA silencing renal clear cell carcinoma cell line Caki-1 was established and confirmed by measuring the expression level of CIP2A using qRT-PCR. As shown in Fig. 4a, CIP2A silencing significantly reduced the expression level of VEGF and phosphorylation level of Akt $(P<0.05)$. However, treatment with Akt activator SC79 (10 $\mu \mathrm{M}$, Sigma-Aldrich) or VEGF (10 nM) for an hour showed no significant effects on expression of CIP2A. Therefore, CIP2A is very likely to serve as an upstream activator of Akt pathway and VEGF.

\section{Akt is likely a positive upstream positive regulator of VEGF in renal clear cell carcinoma cell line Caki-1}

As shown in Fig. 5a, treatment with Akt activator SC79 (10 $\mu \mathrm{M}$, Sigma-Aldrich) for an hour significantly increased the expression level of VEGF in renal clear cell carcinoma cell line Caki-1 $(P<0.05)$. In contrast, treatment with VEGF showed no significant effects on the

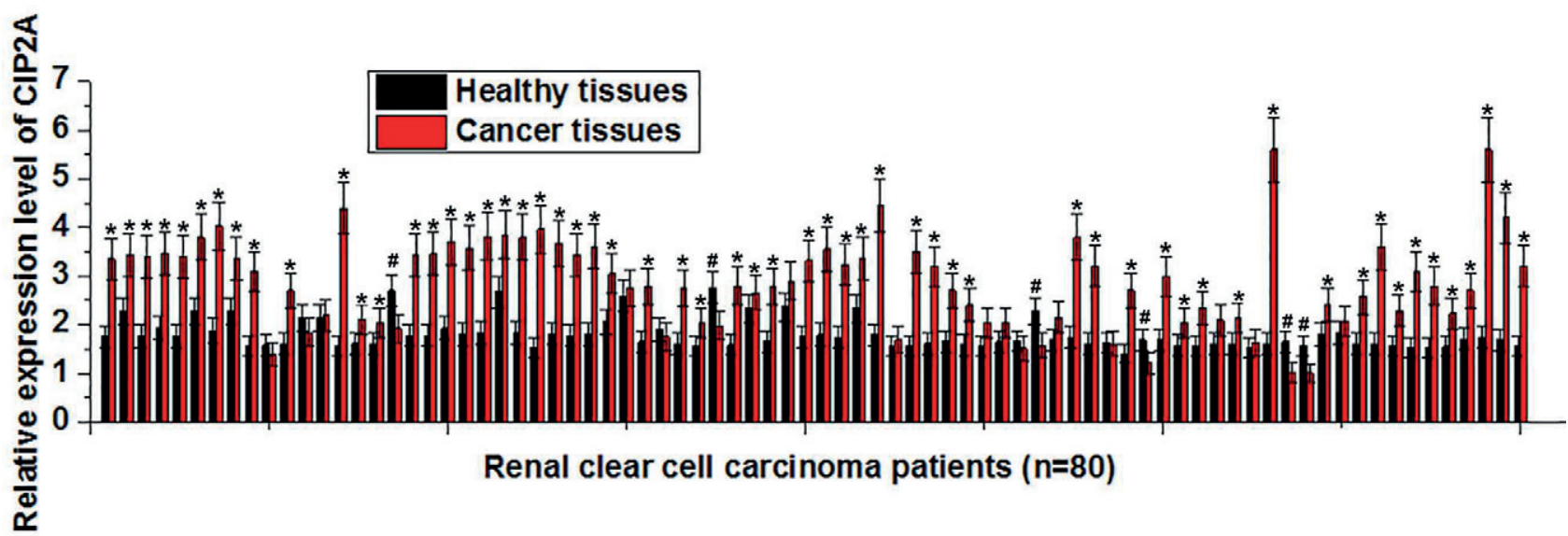

Fig. 1. Expression of CIP2A in tumor tissues and adjacent healthy tissues of 80 patients with renal clear cell carcinoma. Compared with health tissue in the same individual, $P<0.05$; \#, compared with tumor tissue in the same individual, $P<0.05$ 
Table 1. Correlations between basic information and serum levels of CIP2A protein.

\begin{tabular}{|c|c|c|c|c|c|}
\hline Items & Groups & Cases & High-expression & Low-expression & $P$ \\
\hline \multirow{2}{*}{ Gender } & Male & 46 & 21 & 25 & \multirow{2}{*}{0.37} \\
\hline & Female & 34 & 19 & 15 & \\
\hline \multirow{2}{*}{ Age } & $>45$ (years) & 42 & 23 & 19 & \multirow{2}{*}{0.37} \\
\hline & $<45$ (years) & 38 & 17 & 21 & \\
\hline \multirow{2}{*}{ Smoking } & Yes & 44 & 19 & 25 & \multirow{2}{*}{0.17} \\
\hline & No & 36 & 21 & 15 & \\
\hline \multirow{2}{*}{ Drinking } & Yes & 49 & 28 & 21 & \multirow{2}{*}{0.11} \\
\hline & No & 31 & 12 & 19 & \\
\hline
\end{tabular}

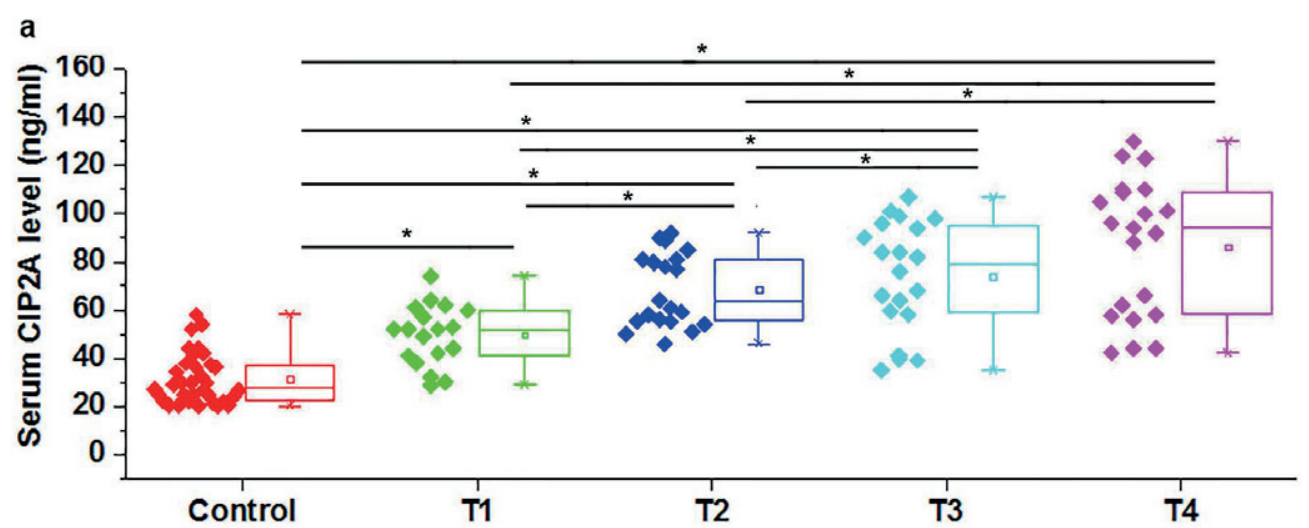

b

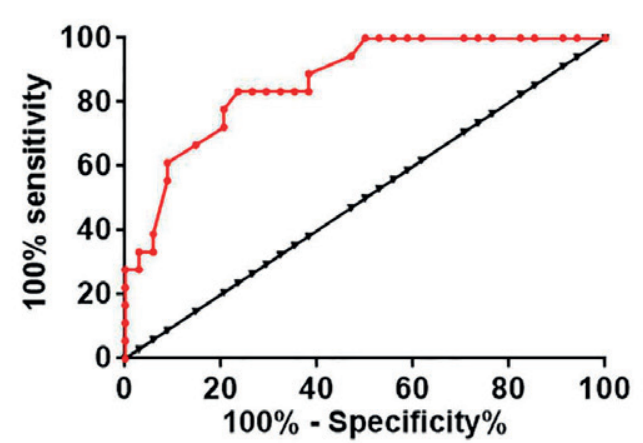

d

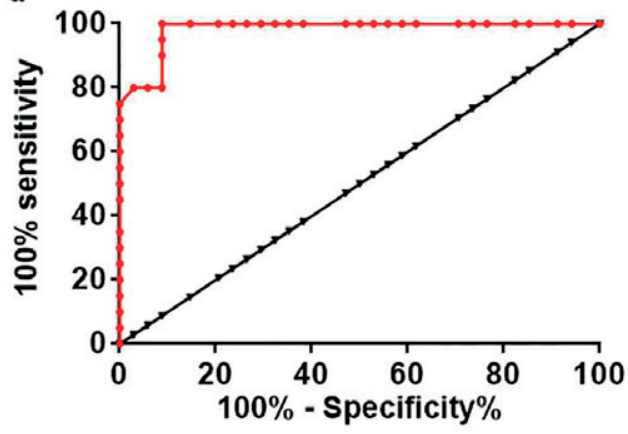

C

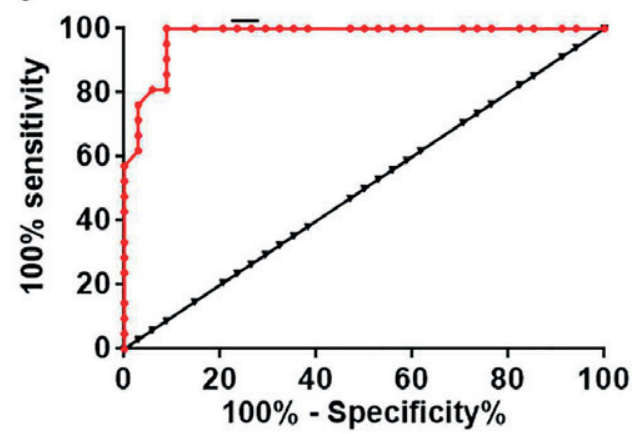

e

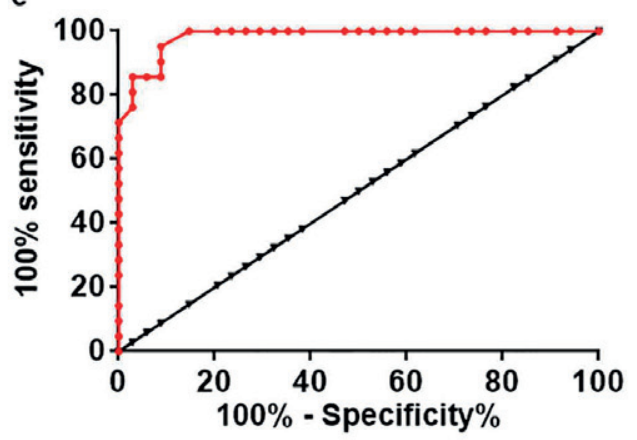

Fig. 2. Serum levels of CIP2A in renal clear cell carcinoma patients and its diagnostic values.

a. Serum levels of CIP2A in patients with different stages of renal clear cell carcinoma; b. ROC curve analysis of the diagnostic value of serum CIP2A for patients with stage $\mathrm{cT} 1$ renal clear cell carcinoma; ROC curve analysis of the diagnostic value of serum CIP2A for patients with stage cT2 renal clear cell carcinoma; c. ROC curve analysis of the diagnostic value of serum CIP2A for patients with stage cT3 renal clear cell carcinoma; ROC curve analysis of the diagnostic value of serum CIP2A for patients with stage cT4 renal clear cell carcinoma.

$* P<0.05$. 


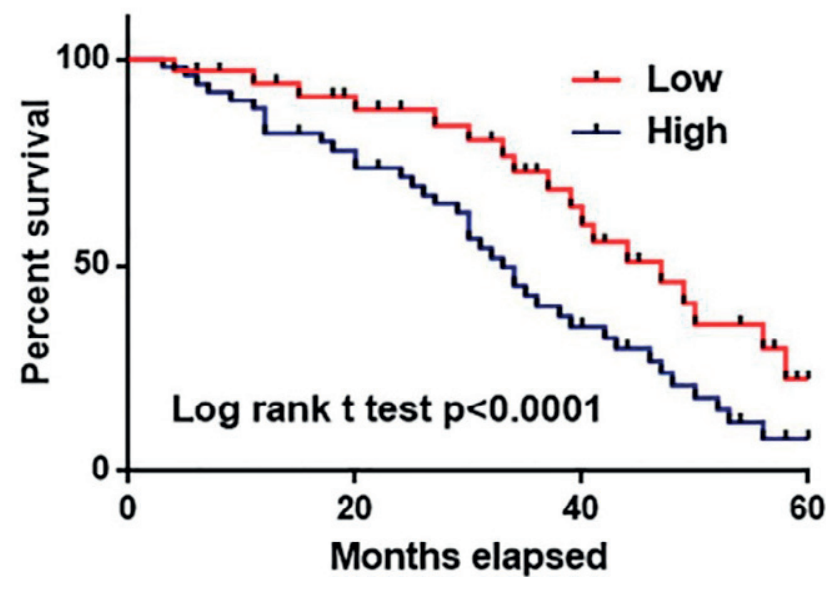

Fig. 3. Comparison of survival curves of patients with high and low serum level of CIP2A.
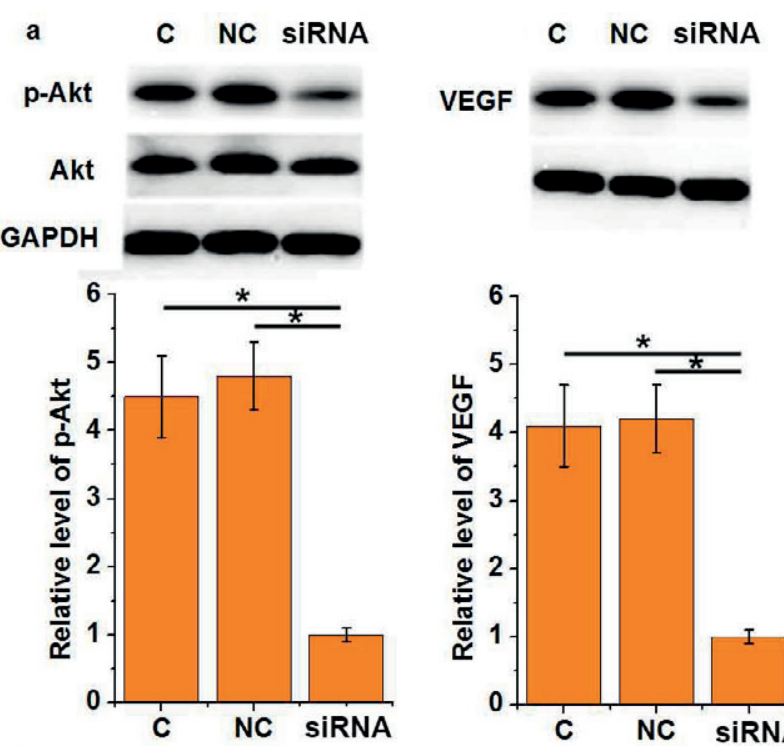

b

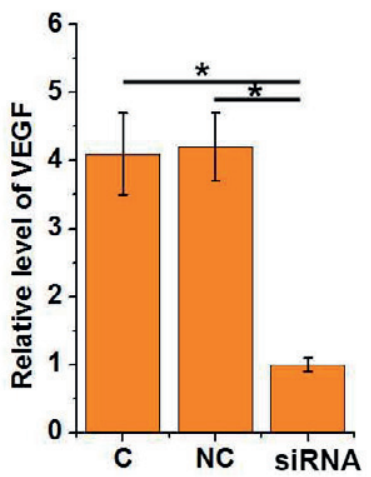

CIP2A

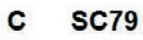

GAPDH
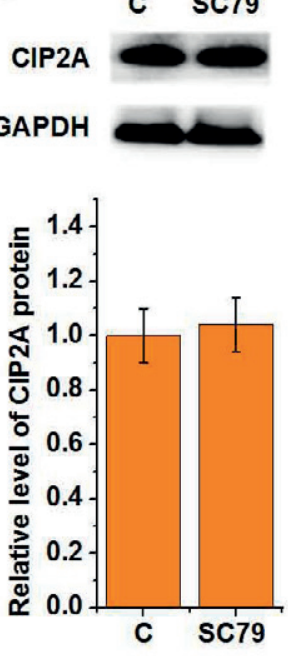

Fig. 4. CIP2A is likely an upstream activator of Akt pathway and VEGF.

a. Effects of CIP2A siRNA silencing on Akt and VEGF; b. Effects of treatment with Akt activator on CIP2A; c. Effects of treatment with VEGF on CIP2A

${ }^{*} P<0.05$.

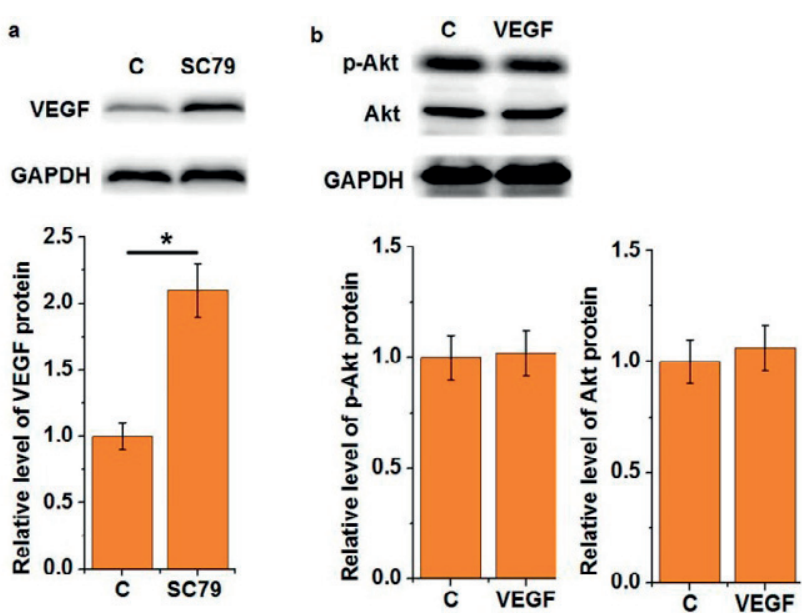

Fig. 5. Akt is likely a positive upstream positive regulator of VEGF in renal clear cell carcinoma cell line Caki-1.

a. Effect of Akt activator on VEGF expression; b. Effect on VEGF treatment on expression and phosphorylation of Akt. $* P<0.05$.

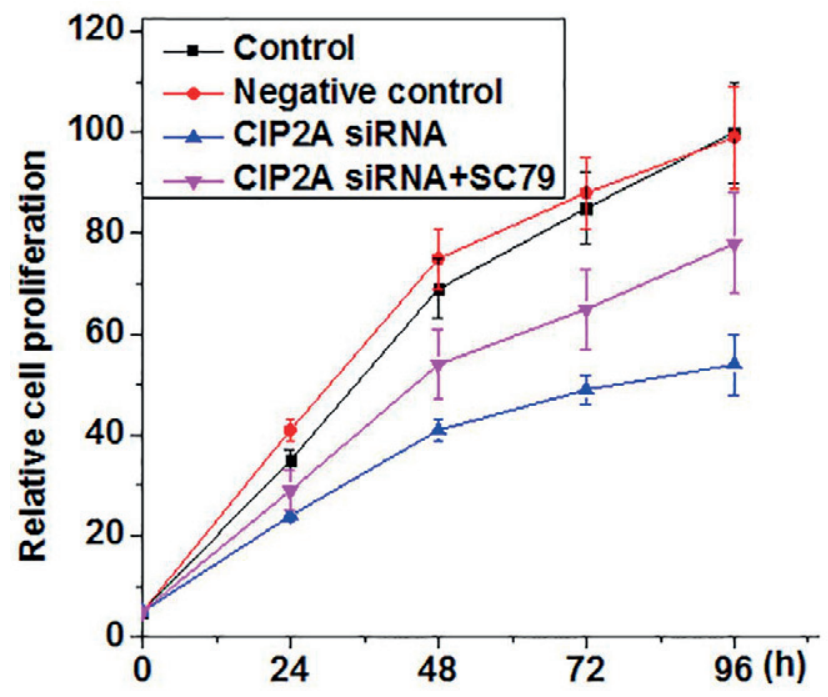

Fig. 6. Effects of CIP2A siRNA silencing on the proliferation of renal clear cell carcinoma cell line Caki-1.

expression and phosphorylation of Akt. Therefore, Akt is very likely to be a positive upstream positive regulator of VEGF in renal clear cell carcinoma cell line Caki-1.

Effects of CIP2A siRNA silencing on the proliferation of renal clear cell carcinoma cell line Caki-1

Compared with control cells, CIP2A silencing significantly inhibited cell proliferation of renal clear cell carcinoma cell line Caki-1 (Fig. 6). In addition, treatment with Akt activator SC79 obviously reduced the inhibitory effect of CIP2A silencing on cell proliferation. The data suggest that CIP2A may regulate renal clear cell carcinoma cell proliferation through the Akt pathway regulation. 


\section{DISCUSSION}

As an oncogene that plays pivotal roles in tumorigenesis, CIP2A is usually overexpressed during the onset, development and progression of different types of malignancies. In the study of non-small cell lung cancer, Dong et al. found that expression level of CIP2A is significantly increased in non-small cell lung cancer cancer patients comparing with that in healthy controls, and patients with high expression level of CIP2A usually have short survival time ${ }^{9}$. Overexpression of CIP2A is also observed in gastric cancer, and the deletion of this gene led to the inhibited senescence, clonogenicity, or differentiation of tumor cells, indicating that CIP2A may serve as target for the treatment of this disease ${ }^{10}$. Upregulation of CIP2A expression is also detected in renal cell carcinoma cells ${ }^{11}$, while its expression in renal clear cell carcinomas is unknown. In our study, significantly higher expression level of CIP2A mRNA in tumor tissues than that in adjacent healthy tissues was observed in most patients with renal clear cell carcinoma. In addition, serum levels of CIP2A were significantly higher in patients with renal clear cell carcinoma than those in healthy controls, and serum levels of CIP2A were increased with the increase of stage of primary tumor. The data suggest that upregulated CIP2A is very likely to be involved in the pathogenesis of renal clear cell carcinoma.

Most patients with renal clear cell carcinoma were diagnosed by CT guided biopsy in advanced stages, which are inappropriate for surgical resection. However surgical resection is the only radical treatment of most types of malignancies including renal clear cell carcinoma ${ }^{12}$. Therefore, early diagnosis and treatment is still the key for the treatment of this disease. Development of cancers is usually accompanied by changes of certain substances in blood, and the monitoring of the blood levels of these substances may provide references for the diagnosis and prognosis of certain malignancies ${ }^{13}$. High expression level of CIP2A predicts poor prognosis of patients with renal cell carcinoma ${ }^{9}$. The results of this study showed that serum CIP2A can be used to effectively distinguish renal clear cell carcinoma patients from normal controls. In addition, patients with higher serum levels of CIP2A showed shorter survival time compared with those with relatively lower serum levels of CIP2A. The data suggest that serum CIP2A protein may serve as a promising diagnostic and prognostic biomarker for renal clear cell carcinoma. Gender ${ }^{14}$, aging ${ }^{15}$, tobacco consumption ${ }^{16}$ and alcohol abuse $^{17}$ affect the expression of certain lncRNAs, which in turn affect the accuracy of $\operatorname{lncRNAs}$ as a biomarker for human diseases. In this study, serum level of CIP2A protein showed no significant correlations with patients' age and gender or living habits including smoking and drinking, indicating that serum CIP2A protein is a reliable biomarker for renal clear cell carcinoma. It is worthwhile to mention that CIP2A is overexpressed in multiple cancers $^{10,11}$. Therefore, multiple biomarkers should be combined in the diagnosis of renal clear cell carcinoma to improve the specificity.
It has been reported that CIP2A regulated Akt signaling pathway to participate in various types of human malignancies, such as lung cancer ${ }^{18}$, hepatocellular carcinoma $^{19}$ and head and neck squamous cell carcinoma ${ }^{20}$. The activation of Akt prevents cancer cell apoptosis induced by chemotherapty ${ }^{19,20}$, resulting in poor treatment outcomes. Akt signaling is involved in the regulation of VEGF expression ${ }^{21}$, which is pivotal for the vascularization of tumor tissues ${ }^{22}$. In this study, our results suggest that CIP2A is very likely to serve as an upstream activator of Akt pathway, and Akt promotes the expression of VEGF in its upstream in renal clear cell carcinoma cells. It is known that CIP2A promotes the proliferation of certain types of tumor cells ${ }^{23}$. In this study, CIP2A siRNA silencing decreased the proliferation rate of renal clear cell carcinoma cells, while treatment with Akt activator significantly reduced the inhibitory effects of CIP2A siRNA silencing on the proliferation of renal clear cell carcinoma cells. The data suggest that downregulation of CIP2A can inhibit cancer cell proliferation and vascularization in renal clear cell carcinoma through inactivation of Akt pathway and its downstream VEGF.

It is worth noting that there is an overlap of serum CIP2A levels of renal clear cell carcinoma patients with healthy individuals, especially for cT1 stage, which will results in inaccurate diagnosis. Therefore, multiple biomarkers should be combined to improve the diagnosis. In addition, only one cell line was included in this study. More cell lines will be included in our future studies to further confirm the conclusions.

\section{CONCLUSIONS}

In conclusion, CIP2A expression level was increased in renal clear cell carcinoma. Serum CIP2A may serve as a promising diagnostic and prognostic biomarker for renal clear cell carcinoma. CIP2A siRNA silencing inhibited tumor cell proliferation. CIP2A siRNA silencing decreased the expression level of VEGF and phosphorylation levels of AKT in renal clear cell carcinoma cells, while AKT activator treatment showed no significant effects on CIP2A expression. Treatment with VEGF showed no significant effect on expression of AKT and VEGF and phosphorylation of Akt. In addition, treatment with Akt activator reduced the inhibitory effect of CIP2A siRNA silencing on tumor cell proliferation. Therefore, we concluded that downregulation of CIP2A can inhibit cancer cell proliferation and vascularization in renal clear cell carcinoma through inactivation of Akt pathway and its downstream VEGF.

Author contributions: HG, YL, TL: did the experiments, collected the data and contributed to the manuscript writing; YC: did the analysis and contributed to the manuscript writing; YM: conceived the study and contributed to the manuscript writing; All authors read and approved the final manuscript.

Conflict of interest statement: None declared. 


\section{REFERENCES}

1. Muglia VF, Prando A. Renal cell carcinoma: histological classification and correlation with imaging findings. Radiol Bras 2015;48(3):16674.

2. Hsieh JJ, Purdue MP, Signoretti S, Swanton C, Albiges L, Schmidinger M, Heng DY, Larkin J, Ficarra V. Renal cell carcinoma. Nat Rev Dis Primers 2017;3:17009.

3. Rini BI, Campbell SC, Escudier B. Renal cell carcinoma. Lancet 2009;373(9669):1119-32.

4. Randall JM, Millard F, Kurzrock R. Molecular aberrations, targeted therapy, and renal cell carcinoma: current state-of-the-art. Cancer and Metastasis Rev 2014;33(4):1109-24.

5. Sato $Y$, Yoshizato T, Shiraishi $Y$, Maekawa S, Okuno $Y$, Kamura $T$, Shimamura T, Sato-Otsubo A, Nagae G, Suzuki H, Nagata Y, Yoshida K, Kon A, Suzuki Y, Chiba K, Tanaka H, Niida A, Fujimoto A, Tsunoda T, Morikawa T, Maeda D, Kume H, Sugano S, Fukayama M, Aburatani $\mathrm{H}$, Sanada M, Miyano S, Homma Y, Ogawa S. Integrated molecular analysis of clear-cell renal cell carcinoma. Nat Genet 2013;45(8):860

6. Duns G, van den Berg E, van Duivenbode I, Osinga J, Hollema $H_{\text {, }}$ Hofstra RM, Kok K. Histone methyltransferase gene SETD2 is a novel tumor suppressor gene in clear cell renal cell carcinoma. Cancer Res 2010;0008-5472. CAN-10-0120.

7. Gossage L, Murtaza M, Slatter AF, Lichtenstein CP, Warren A, Haynes B, Marass F, Roberts I, Shanahan SJ, Claas A, Dunham A, May AP, Rosenfeld N, Forshew T, Eisen T. Clinical and pathological impact of VHL, PBRM1, BAP1, SETD2, KDM6A, and JARID1c in clear cell rena cell carcinoma. Genes Chromosomes Cancer 2014;53(1):38-51.

8. Liu H, Qiu H, Song Y, Liu Y, Wang H, Lu M, Deng M, Gu Y, Yin J, Luo K, Zhang Z, Jia X, Zheng G, He Z. Cip2a promotes cell cycle progression in triple-negative breast cancer cells by regulating the expression and nuclear export of p27Kip1. Oncogene 2017;36(14):1952.

9. Dong QZ, Wang Y, Dong XJ, Li ZX, Tang ZP, Cui QZ, Wang EH. CIP2A is overexpressed in non-small cell lung cancer and correlates with poor prognosis. Ann Surg Oncol 2011;18(3):857-65.

10. Li W, Ge Z, Liu C, Liu Z, Bjorkholm M, Jia J, Xu D. CIP2A is overexpressed in gastric cancer and its depletion leads to impaired clonogenicity, senescence, or differentiation of tumor cells. Clin Cancer Res 2008;14(12):3722-8.

11. Ren J, Li W, Yan L, Jiao W, Tian S, Li D, Tang Y, Gu G, Liu H, Xu Z. Expression of CIP2A in renal cell carcinomas correlates with tumour invasion, metastasis and patients' survival. Br J Cancer 2011;105(12):1905.

12. Motzer RJ, Escudier B, Oudard S, Hutson TE, Porta C, Bracarda S, Grunwald V, Thompson JA, Figlin RA, Hollaender N, Urbanowitz
G, Berg WJ, Kay A, Lebwohl D, Ravaud A, RECORD-1 Study Group. Efficacy of everolimus in advanced renal cell carcinoma: a double-blind, randomised, placebo-controlled phase III trial. Lancet 2008:372(9637):449-56.

13. Hori SS, Gambhir SS. Mathematical model identifies blood biomarker-based early cancer detection strategies and limitations. Sci Transl Med 2011;3(109):109ra116

14. Li X, Liu R, Yang J, Sun L, Zhang L, Jiang Z, Puri P, Gurley EC, La G, Tang Y, Huang Z, Pandak WM, Hylemon PB, Zhou H. The role of long noncoding RNA H19 in gender disparity of cholestatic liver injury in multidrug resistance 2 gene knockout mice. Hepatology 2017;66(3):869-84.

15. Bianchessi V, Badi I, Bertolotti M, Nigro P, D'Alessandra Y, Capogrossi MC, Zanobini M, Pompilio G, Raucci A, Lauri A. The mitochondrial IncRNA ASncmtRNA-2 is induced in aging and replicative senescence in Endothelial Cells. J Mol Cell Cardiol 2015;81:62-70.

16. Lu L, Xu H, Luo F, Liu X, Lu X, Yang Q, Xue J, Chen C, Shi L, Liu O Epigenetic silencing of miR-218 by the IncRNA CCAT1, acting via BMI1, promotes an altered cell cycle transition in the malignant transformation of HBE cells induced by cigarette smoke extract. Toxicol Appl Pharmacol 2016;304:30-41.

17. Soares do Amaral N, Cruz e Melo N, de Melo Maia B, Malagoli Rocha R. Noncoding RNA profiles in tobacco-and alcohol-associated diseases. Genes 2016;8(1):6.

18. Lei N, Peng B, ZhANG JY. CIP2A regulates cell proliferation via the AKT signaling pathway in human lung cancer. Oncol Rep 2014;32(4):1689-94.

19. Chen KF, Liu CY, Lin YC, Yu HC, Liu TH, Hou DR, Chen PJ, Cheng AL. CIP2A mediates effects of bortezomib on phospho-Akt and apoptosis in hepatocellular carcinoma cells. Oncogene 2010;29(47):6257.

20. Lin YC, Chen KC, Chen CC, Cheng AL, Chen KF. CIP2A-mediated Akt activation plays a role in bortezomib-induced apoptosis in head and neck squamous cell carcinoma cells. Oral Oncol 2012;48(7):585-93.

21. Huang Y, Mao Y, Li H, Shen G, Nan G. Knockdown of Nrf2 inhibits angiogenesis by downregulating VEGF expression through PI3K/ Akt signaling pathway in cerebral microvascular endothelial cells under hypoxic conditions. Biochem Cell Biol 2018;96(4):475-82. doi: 10.1139/bcb-2017-0291

22. Grun D, Adhikary G, Eckert RL. VEGF-A acts via neuropilin-1 to enhance epidermal cancer stem cell survival and formation of aggressive and highly vascularized tumors. Oncogene 2016; 35(33):4379.

23. Gao F, XU T, Wang X, Zhong S, Chen S, Zhang M, Zhang X, Shen Y, Wang X, Xu C, Shen Z. CIP2A mediates fibronectin-induced bladder cancer cell proliferation by stabilizing $\beta$-catenin. J Exp Clin Cancer Res 2017;36(1):70. 